\title{
Neutrinoful universe
}

\author{
Tetsutaro Higaki, ${ }^{a}$ Ryuichiro Kitano ${ }^{a, b}$ and Ryosuke Sato ${ }^{a}$ \\ ${ }^{a}$ Institute of Particle and Nuclear Studies, \\ High Energy Accelerator Research Organization (KEK), \\ Tsukuba 305-0801, Japan \\ ${ }^{b}$ The Graduate University for Advanced Studies (Sokendai), \\ Tsukuba 305-0801, Japan \\ E-mail: thigaki@post.kek.jp, ryuichiro.kitano@kek.jp, \\ rsato@post.kek.jp
}

ABSTRACT: The Standard Model of particle physics fails to explain the important pieces in the standard cosmology, such as inflation, baryogenesis, and dark matter of the Universe. We consider the possibility that the sector to generate small neutrino masses is responsible for all of them; the inflation is driven by the Higgs field to break $B-L$ gauge symmetry which provides the Majorana masses to the right-handed neutrinos, and the reheating process by the decay of the $B-L$ Higgs boson supplies the second lightest right-handed neutrinos whose $\mathrm{CP}$ violating decays produce $B-L$ asymmetry, $\grave{a} l a$, leptogenesis. The lightest right-handed neutrinos are also produced by the reheating process, and remain today as the dark matter of the Universe. In the minimal model of the inflaton potential, one can set the parameter of the potential by the data from CMB observations including the BICEP2 and the Planck experiments. In such a scenario, the mass of the dark matter particle is predicted to be of the order of $\mathrm{PeV}$. We find that the decay of the $\mathrm{PeV}$ right-handed neutrinos can explain the high-energy neutrino flux observed at the IceCube experiments if the lifetime is of the order of $10^{28} \mathrm{~s}$.

Keywords: Cosmology of Theories beyond the SM, Neutrino Physics

ArXiv EPRINT: 1405.0013 


\section{Contents}

1 Introduction 1

2 Model 2

3 Inflation with the $B-L$ Higgs field $\quad 6$

4 Reheating by the inflaton decay $\quad 7$

4.1 Leptogenesis 8

$\begin{array}{ll}4.2 & \text { Dark matter abundance }\end{array}$

$5 \quad$ PeV neutrinos as a signal of decaying $N_{1} \quad 9$

$\begin{array}{ll}5.1 \text { The branching fractions of } N_{1} & 10\end{array}$

$\begin{array}{ll}5.2 \text { Neutrino flux from decay of } N_{1} & 11\end{array}$

$\begin{array}{llr}6 & \text { Summary } & 14\end{array}$

\section{Introduction}

Various cosmological observations are telling us that the Standard Model of particle physics needs some extension. The observation of the cosmic microwave background (CMB) and its anisotropy strongly supports the inflationary cosmology [1-3], which requires a process to generate the Standard Model particles after the inflation era. The particle-antiparticle asymmetry should also be generated after or during the reheating process. Also, the dark matter of the Universe must also be produced in the course of the cosmological history. The Standard Model should be extended to accommodate the inflation, baryogenesis and dark matter of the Universe.

One of the clues towards the mysteries of the Universe may be the finite neutrino masses, which are another evidence to go beyond the Standard Model. Once three kinds of right-handed neutrinos are introduced in the same way as other fermions, the global $U(1)_{B-L}$ symmetry becomes non-anomalous, and thus can be promoted to a Higgsed gauge symmetry. It seems that all the ingredients to accommodate the realistic cosmology are present in this $U(1)_{B-L}$ extended Standard Model.

The inflation can be driven by the Higgs field to break $U(1)_{B-L}$ gauge symmetry [4, $5]$ by assuming an appropriate form of the potential based on the idea of the chaotic inflation [6]. After the inflation, the $B-L$ Higgs field oscillates about the minimum of the potential where $U(1)_{B-L}$ is broken. The spontaneous breaking of the $B-L$ symmetry can give Majorana masses to the right-handed neutrinos through the Yukawa coupling, explaining the smallness of the neutrino masses by the seesaw mechanism [7-12]. The very 
same coupling allows the decay of the inflaton oscillation into the right-handed neutrinos to reheat the Universe. The subsequent decay of the right-handed neutrinos can provide the baryon asymmetry of the Universe by the leptogenesis mechanism [13]. The lightest right-handed neutrino should also be produced by the inflaton decay. If it is long-lived, this non-thermal component is a good candidate of the dark matter of the Universe.

There have been other minimalistic approaches to the connection between particle physics and cosmology. An realistic model with the minimal particle content has been constructed in ref. [14], where the inflaton and the dark matter particle are both introduced as new scalar fields. The possibility of the inflaton as the Higgs-like field, thus playing important roles both in particle physics and cosmology, has been considered in refs. [1529]. The dark matter of the Universe as the right-handed neutrino has also been considered in refs. [30-39] where the mass range of $\mathcal{O}(\mathrm{keV})$ are assumed.

In this paper, we consider the $U(1)_{B-L}$ extended Standard Model which covers the shortages in the Standard Model including the small neutrino masses as well as cosmological observations. We find that this minimalistic scenario is consistent with various observations such as tensor-to-scalar ratio, spectral index of the CMB fluctuations, the neutrino masses, baryon asymmetry of the Universe, and the energy density of the dark matter. We find, in the case where the reheating process is dominated by the decay of inflaton into the second lightest right-handed neutrinos, the mass of the dark matter particle is predicted to be of the order of $\mathrm{PeV}$.

Since there is no reason to assume that the dark matter particle, the lightest righthanded neutrino, to be absolutely stable, we expect the decay of the dark matter to happen occasionally somewhere in the Universe. Through the dimension-four Yukawa interactions, the main decay mode would be into a lepton and a $W$ boson, or a neutrino and a $Z / h$ boson. We demonstrate that the PeV neutrino events found at the IceCube experiment $[40,41]$ can be explained by the decaying right-handed neutrinos if the lifetime is of the order of $10^{28} \mathrm{~s} .{ }^{1}$

In the following sections, based on the above scenario with $U(1)_{B-L}$ extended Standard Model, we discuss the neutrino flavor structure, an inflation model with the $B-L$ Higgs, the non-thermal leptogenesis, the dark matter abundance produced by the decay of the inflaton, and the signals of decaying right-handed neutrinos at the IceCube experiment.

\section{$2 \quad$ Model}

We extend the gauge group of the Standard Model into,

$$
S U(3)_{c} \times S U(2)_{L} \times U(1)_{Y} \times U(1)_{B-L}
$$

and introduce the right-handed neutrinos, $N_{i}(i=1,2,3)$, and the $U(1)_{B-L}$ Higgs field $\phi_{B-L}$ which is neutral under the Standard Model gauge group and has charge -2 under $U(1)_{B-L}$. The $U(1)_{B-L}$ symmetry is gauged, and thus the spontaneous breaking of $U(1)_{B-L}$ would not leave the massless Nambu-Goldstone boson. The following interaction

\footnotetext{
${ }^{1}$ See, e.g., refs. $[42,43]$ for studies on $\mathrm{PeV}$ decaying dark matter.
} 
terms are added to the Standard Model:

$$
\mathcal{L}_{\text {int }}=y_{\nu}^{i j} \bar{N}_{i} P_{L}\left(\ell_{j} \cdot \tilde{H}\right)+\text { h.c. }+\frac{\lambda_{i}}{2} \phi_{B-L} \bar{N}_{i} P_{L} N_{i}^{c}+\text { h.c. },
$$

where $\ell_{i}$ and $N_{i}$ are four-component Weyl fermions, i.e., $P_{L} \ell_{i}=\ell_{i}, P_{R} N_{i}=N_{i}$. The coupling constant $\lambda_{i}$ can be taken to be real and positive without loss of generality, and the components of $y_{\nu}^{i j}$, in general, are complex valued. The potential terms for $\phi_{B-L}$ field can be written as,

$$
V(\phi)=\frac{\kappa}{4}\left(\left|\phi_{\mathrm{B}-\mathrm{L}}\right|^{2}-v_{B-L}^{2}\right)^{2}=\frac{\kappa v_{B-L}^{4}}{4}\left(\frac{\left|\phi_{\mathrm{B}-\mathrm{L}}\right|^{2}}{v_{B-L}^{2}}-1\right)^{2} .
$$

There can also be an interaction term such as,

$$
\mathcal{L}_{\phi H}=\kappa^{\prime}\left|\phi_{B-L}\right|^{2}|H|^{2} .
$$

For $v_{B-L} \gtrsim 5 M_{\mathrm{Pl}}$ which we assume later, the coupling constant $\kappa^{\prime}$ is extremely small if we demand this term would not contribute significantly to the Higgs potential.

The spontaneous breaking of $U(1)_{B-L}$ by $\left\langle\phi_{B-L}\right\rangle=v_{B-L}$ generates masses of $N_{i}$ :

$$
M_{i}=\lambda_{i} v_{B-L} .
$$

The neutrino masses are, in turn, generated by the seesaw mechanism:

$$
m_{\nu}^{i j}=y_{\nu}^{k i} M_{k}^{-1} y_{\nu}^{k j}\langle H\rangle^{2} .
$$

We assume that the lightest right-handed neutrino, $N_{1}$, to be long-lived, and it serves as the dark matter of the Universe. That means,

$$
\left|y_{\nu}^{1 i}\right| \ll 1
$$

As we will see in section 5 , in the scenario where the $\mathrm{PeV}$ neutrino events at the IceCube experiment to be explained by the decay of $N_{1}$, the lifetime of $N_{1}$ has to be around $10^{28} \mathrm{~s}$. This lifetime corresponds to $y_{\nu}^{1 i} \sim 10^{-29}$. In fact, this model has various unexplained small numbers such as the Higgs mass parameter, the $\theta$ parameter in QCD, the cosmological constant, $\kappa^{\prime}, \kappa$ as well as $y_{\nu}^{1 i}$. Although we do not look for particular reasons for such small numbers here, a very small $y_{\nu}^{1 i}$ is somewhat special since it can be protected by a $Z_{2}$ symmetry, $N_{1} \leftrightarrow-N_{1}$. If such a symmetry is only violated by some non-perturbative effects of gauge or gravity interactions at high scales, the size may be understood as a natural value. ${ }^{2}$ In such a scenario, it is likely that the non-perturbative effects respect the flavor symmetry, and thus the effective operator to break the $Z_{2}$ symmetry, for example, takes the form of

$$
\mathcal{L}_{N P}=\frac{1}{\Lambda^{14}}\left(\ell_{1} \cdot \ell_{2}\right)\left(\ell_{2} \cdot \ell_{3}\right)\left(\ell_{3} \cdot \ell_{1}\right) e_{1}^{c} e_{2}^{c} e_{3}^{c} N_{1}^{c} N_{2}^{c} N_{3}^{c}+\text { h.c. }
$$

\footnotetext{
${ }^{2}$ The quantum theory of gravity may give natural ground for such considerations [44-47].
} 


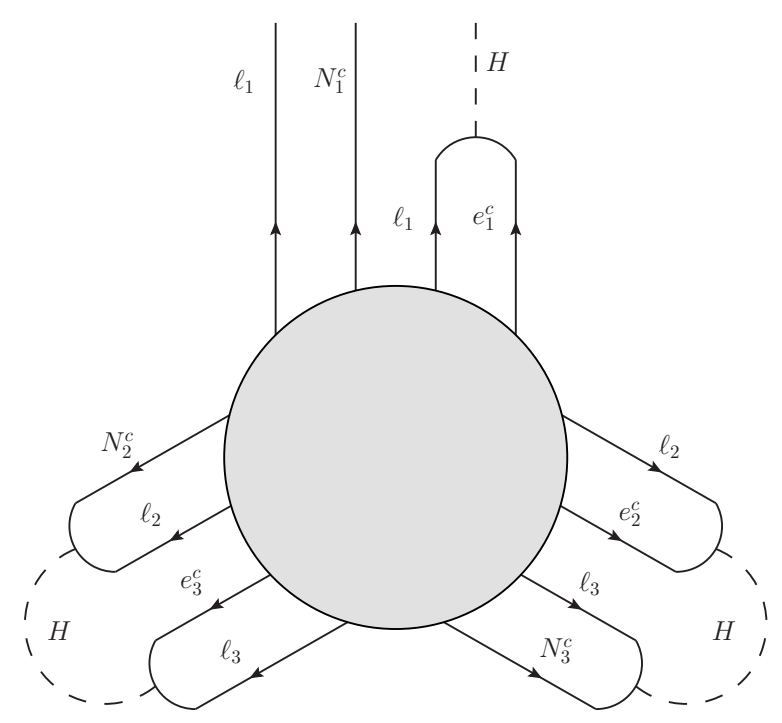

Figure 1. One of the diagrams generating $y_{\nu}^{1 k}$ with the interaction in eq. (2.7).

Here, $\Lambda$ is expected to be the scale which characterizes the non-perturbative effects such as, $\mu e^{-8 \pi^{2} / g^{2}(\mu)}$, in the case of a gauge theory. This is analogous to the interaction considered in QCD $[48,49]$. Together with the Yukawa interactions of the charged lepton sector $y_{e}^{i j}$ in the Standard Model and $y_{\nu}^{\alpha i}(\alpha=2,3)$ in eq. $(2.1), y_{\nu}^{1 i}$ is generated as in the diagram in figure 1:

$$
y_{\nu}^{1 k} \propto\left(\operatorname{det} y_{e}\right) \epsilon^{i j k} y_{\nu}^{2 i} y_{\nu}^{3 j}
$$

One can also consider interactions such as $\mathcal{L}_{N P}=\left(q_{1} \cdot \ell_{2}\right)\left(q_{2} \cdot \ell_{3}\right)\left(q_{3} \cdot \ell_{1}\right) d_{1}^{c} d_{2}^{c} d_{3}^{c} N_{1}^{c} N_{2}^{c} N_{3}^{c} / \Lambda^{14}$. From this operator, we obtain $y_{\nu}^{1 k} \propto\left(\operatorname{det} y_{d}\right) \epsilon^{i j k} y_{\nu}^{2 i} y_{\nu}^{3 j}$. In any case, the flavor symmetry implies an interesting proportionality:

$$
y_{\nu}^{1 k} \propto \epsilon^{i j k} y_{\nu}^{2 i} y_{\nu}^{3 j}
$$

We will see in section 5 that if this type of contribution is dominated, the branching ratio of the $N_{1}$ decay is directly related to the neutrino mixing parameters. By introducing a small parameter $c$, eq. (2.9) is explicitly written as

$$
y_{\nu}^{1 e}=c\left(y_{\nu}^{2 \mu} y_{\nu}^{3 \tau}-y_{\nu}^{3 \mu} y_{\nu}^{2 \tau}\right), \quad y_{\nu}^{1 \mu}=c\left(y_{\nu}^{2 \tau} y_{\nu}^{3 e}-y_{\nu}^{3 \tau} y_{\nu}^{2 e}\right), \quad y_{\nu}^{1 \tau}=c\left(y_{\nu}^{2 e} y_{\nu}^{3 \mu}-y_{\nu}^{3 e} y_{\nu}^{2 \mu}\right)
$$

Because of tiny $y_{\nu}^{1 \ell}$ 's, $N_{1}$ provides very little contribution to the neutrino masses. In this case, the neutrino sector is essentially that of the model with only two right-handed neutrinos $[50,51]$. Here, we define the following Yukawa matrix $\tilde{y}$ and mass matrix $\tilde{M}$ :

$$
\tilde{y}=\left(\begin{array}{ccc}
y_{\nu}^{2 e} & y_{\nu}^{2 \mu} & y_{\nu}^{2 \tau} \\
y_{\nu}^{3 e} & y_{\nu}^{3 \mu} & y_{\nu}^{3 \tau}
\end{array}\right), \quad \tilde{M}=\left(\begin{array}{cc}
M_{2} & 0 \\
0 & M_{3}
\end{array}\right) .
$$


Neutrino masses are given by,

$$
m_{\nu} \equiv \operatorname{diag}\left(m_{1}, m_{2}, m_{3}\right)=\left(U_{\mathrm{PMNS}}^{T} \tilde{y}^{T} \tilde{M}^{-1} \tilde{y} U_{\mathrm{MNS}}\right)\langle H\rangle^{2},
$$

where $U_{\text {MNS }}$ is the Pontecorvo-Maki-Nakagawa-Sakata (PMNS) matrix [52, 53]:

$$
\begin{aligned}
U_{\mathrm{PMNS}}=\left(\begin{array}{ccc}
U_{e 1} & U_{e 2} & U_{e 3} \\
U_{\mu 1} & U_{\mu 2} & U_{\mu 3} \\
U_{\tau 1} & U_{\tau 2} & U_{\tau 3}
\end{array}\right)= & \left(\begin{array}{ccc}
c_{12} c_{13} & s_{12} c_{13} & s_{13} e^{-i \delta} \\
-s_{12} c_{23}-c_{12} s_{23} s_{13} e^{i \delta} & c_{12} c_{23}-s_{12} s_{23} s_{13} e^{i \delta} & s_{23} c_{13} \\
s_{12} s_{23}-c_{12} c_{23} s_{13} e^{i \delta} & -c_{12} s_{23}-s_{12} c_{23} s_{13} e^{i \delta} & c_{23} c_{13}
\end{array}\right) \\
& \times \operatorname{diag}\left(1, e^{i \alpha / 2}, 1\right) .
\end{aligned}
$$

Eq. (2.12) tells us that the lightest neutrino is massless (up to $O\left(\left(y_{\nu}^{1 i}\right)^{2}\right)$ contributions) because the rank of $\tilde{y}$ and $\tilde{M}$ is two. There is only one Majorana phase in eq. (2.13) in this effectively two-generation model. We can parametrize $\tilde{y}$ which satisfies eq. (2.12) by using a $3 \times 2$ complex matrix $R[54,55]$ :

$$
\tilde{y}=\frac{1}{\langle H\rangle} \tilde{M}^{1 / 2} R m_{\nu}^{1 / 2} U_{\mathrm{PMNS}}^{\dagger}
$$

where $R$ can be expressed in terms of a complex parameter $z$,

$$
R=\left(\begin{array}{ccc}
0 & \cos z & -\sin z \\
0 & \sin z & \cos z
\end{array}\right),
$$

for normal hierarchy, and,

$$
R=\left(\begin{array}{ccc}
\cos z & -\sin z & 0 \\
\sin z & \cos z & 0
\end{array}\right)
$$

for inverted hierarchy.

By using the above parametrization and eqs. (2.10), (2.14), we can determine the structure of the Yukawa coupling $y_{\nu}$. For normal hierarchy, we obtain,

$$
\begin{aligned}
y_{\nu}^{1 \ell} & =c \frac{\sqrt{M_{2} M_{3} m_{2} m_{3}}}{\langle H\rangle^{2}} \operatorname{det} U_{\mathrm{PMNS}}^{*} \times U_{\ell 1}, \\
y_{\nu}^{2 \ell} & =\frac{\sqrt{M_{2}}}{\langle H\rangle}\left(\sqrt{m_{2}} U_{\ell 2}^{*} \cos z-\sqrt{m_{3}} U_{\ell 3}^{*} \sin z\right), \\
y_{\nu}^{3 \ell} & =\frac{\sqrt{M_{3}}}{\langle H\rangle}\left(\sqrt{m_{2}} U_{\ell 2}^{*} \sin z+\sqrt{m_{3}} U_{\ell 3}^{*} \cos z\right) .
\end{aligned}
$$

For inverted hierarchy,

$$
\begin{aligned}
y_{\nu}^{1 \ell} & =c \frac{\sqrt{M_{2} M_{3} m_{1} m_{3}}}{\langle H\rangle^{2}} \operatorname{det} U_{\mathrm{PMNS}}^{*} \times U_{\ell 3}, \\
y_{\nu}^{2 \ell} & =\frac{\sqrt{M_{2}}}{\langle H\rangle}\left(\sqrt{m_{1}} U_{\ell 1}^{*} \cos z-\sqrt{m_{2}} U_{\ell 2}^{*} \sin z\right), \\
y_{\nu}^{3 \ell} & =\frac{\sqrt{M_{3}}}{\langle H\rangle}\left(\sqrt{m_{1}} U_{\ell 1}^{*} \sin z+\sqrt{m_{2}} U_{\ell 2}^{*} \cos z\right) .
\end{aligned}
$$

Here, we used the unitarity of $U_{\mathrm{PMNS}}$ for the calculation of $y_{\nu}^{1 \ell}$. These structures are important for the discussion of the flavor of the decay products of $N_{1}$, We will discuss their effects on the energy spectrum of the neutrino flux from the decay of $N_{1}$ in section 5 . 


\section{Inflation with the $B-L$ Higgs field}

In this section, we consider an inflation model with the $B-L$ Higgs field. The potential for $\phi_{B-L}$ in eq. (2.2) can drive inflation of the Universe. By defining $\phi=\sqrt{2}\left|\phi_{B-L}\right|$, the potential is recast in the form of,

$$
V(\phi)=\Lambda^{4}\left(\frac{\phi^{2}}{\mu^{2}}-1\right)^{2}
$$

where $\mu^{2}=2 v_{B-L}^{2}$ and $\Lambda^{4}=\kappa v_{B-L}^{4} / 4$, and we define $\mu>0$. The phase direction can be gauged away.

The inflaton field can slow roll when $\mu \gg M_{\mathrm{Pl}}$, either from the $|\phi|>\mu$ or $|\phi|<\mu$ region towards the minimum at $\phi=\mu$. In both cases, the slow-roll parameters at $\phi=\phi_{0}$ are given by [56],

$$
\epsilon=\frac{M_{\mathrm{Pl}}^{2}}{2}\left(\frac{V^{\prime}}{V}\right)^{2}=\frac{M_{\mathrm{Pl}}^{2}}{2 \mu^{2}}\left(\frac{\frac{4 \phi_{0}}{\mu}}{\frac{\phi_{0}^{2}}{\mu^{2}}-1}\right)^{2}, \quad \eta=M_{\mathrm{Pl}}^{2} \frac{V^{\prime \prime}}{V}=\frac{4 M_{\mathrm{Pl}}^{2}}{\mu^{2}} \frac{\frac{3 \phi_{0}^{2}}{\mu^{2}}-1}{\left(\frac{\phi_{0}^{2}}{\mu^{2}}-1\right)^{2}} .
$$

The field value $\phi_{0}$ at the pivot scale $k_{0}=0.002 \mathrm{Mpc}^{-1}$ is expressed in terms of the number of $e$-folds $N$ :

$$
N \simeq \frac{1}{M_{\mathrm{Pl}}^{2}} \int_{\phi_{\mathrm{end}}}^{\phi_{0}} \frac{V}{V^{\prime}} d \phi=\frac{\mu}{M_{\mathrm{Pl}}^{2}} \int_{\phi_{\mathrm{end}}}^{\phi_{0}} \frac{\frac{\phi^{2}}{\mu^{2}}-1}{\frac{4 \phi}{\mu}} d \phi,
$$

where the field value at the end of the inflation, $\phi_{\text {end }}$, is obtained from,

$$
\left.1 \simeq M_{\mathrm{Pl}}^{2}\left(\frac{V^{\prime}}{V}\right)^{2}\right|_{\phi=\phi_{\mathrm{end}}}=\frac{M_{\mathrm{Pl}}^{2}}{\mu^{2}}\left(\frac{\frac{4 \phi_{\mathrm{end}}}{\mu}}{\frac{\phi_{\mathrm{end}}^{2}}{\mu^{2}}-1}\right)^{2} .
$$

The tensor-to-scalar ratio, $r$, and the spectral index, $n_{s}$, is expressed in terms of the slowroll parameters as,

$$
r=16 \epsilon, \quad n_{s}=1-6 \epsilon+2 \eta .
$$

The Planck normalization sets the overall scale [57],

$$
\left.\left(\frac{V}{\epsilon}\right)^{1 / 4}\right|_{\phi_{0}}=6.4 \times 10^{16} \mathrm{GeV}
$$

and the observed spectral index is given by,

$$
n_{s}=0.9603 \pm 0.0073 .
$$

The results from the BICEP2 experiment prefer,

$$
r=0.20_{-0.05}^{+0.07}, \quad V^{1 / 4}=2.0 \times 10^{16} \mathrm{GeV} \cdot\left(\frac{r}{0.16}\right)^{1 / 4}
$$





Figure 2. Predictions of the inflation model in the $r-n_{s}$ plane (left) and $m_{\phi}-\mu / M_{\mathrm{Pl}}$ plane (right). The region favored by CMB observations (Planck+WP+highL+BICEP2) [58] are also shown in the left figure. The dark blue region corresponds to the region consistent with the BICEP2 at $1 \sigma$ level whereas the light blue region does to that at $2 \sigma$ level. The left figure is consistent with the result obtained in ref. [5].

when one combines the data from the Planck experiment. ${ }^{3}$ Here, the preferred range of $r$ will be modified to $r=0.16_{-0.05}^{+0.06}$ after subtracting the best available estimate for foreground dust [58].

The predictions for $r$ and $n_{s}$ is shown in figure 2 with varying $\mu$. The region favored by the CMB observations are also shown. We see that for $N=60,|\phi|>\mu$ and $\mu \gtrsim 5 M_{\mathrm{Pl}}$ is favored. The inflaton mass, $m_{\phi}=2 \sqrt{2} \Lambda^{2} / \mu$, as a function of $\mu / M_{\mathrm{Pl}}$ is also shown in figure 2. For $|\phi|>\mu$ and $\mu \gtrsim 5 M_{\mathrm{Pl}}$, we find,

$$
m_{\phi} \sim 10^{13} \mathrm{GeV} .
$$

This value corresponds to a very small value of $\kappa$ such as $\kappa \sim 10^{-12}$ for $\mu \sim 5 M_{\mathrm{Pl}}$. In the following discussion, we fix the inflaton mass at this value, and will see that the correct amount of the baryon asymmetry and the dark matter can be obtained after the decay of the inflaton fields.

\section{Reheating by the inflaton decay}

After the inflation, the decay of $\phi$ can produce the Standard Model particles. The dominant decay mode can either be into two right-handed neutrinos via the interaction term in eq. (2.1) or two Higgs fields (including the Goldstone modes) via the term in eq. (2.3).

\footnotetext{
${ }^{3}$ We will not consider the tension between the data from the Planck satellite $(r<0.11)$ [57] and that from the BICEP2 experiment $(r \sim 0.2)$. The tension can be relaxed if one considers a running spectral index, an extra relativistic component, non-zero neutrino mass [58, 59], an anti-correlation between tensor and scalar modes [60] or between tensor and isocurvature modes [61,62]. See also [63-69] for other solutions.
} 
In the case where the $\phi \rightarrow N_{i} N_{i}$ mode is dominated and for $\lambda_{1} \ll \lambda_{2}$ and $M_{3}>m_{\phi}$ which are justified later, the decay width is given by,

$$
\Gamma_{\phi}=\frac{1}{2} \frac{m_{\phi}}{16 \pi} \frac{M_{2}^{2}}{v_{B-L}^{2}}\left(1-\frac{4 M_{2}^{2}}{m_{\phi}^{2}}\right)^{3 / 2} .
$$

By equating $\Gamma_{\phi}$ with the Hubble parameter $H\left(T_{R}\right)$ at the reheating temperature, $T_{R}$, we obtain,

$$
T_{R} \simeq 2 \times 10^{7} \mathrm{GeV}\left(\frac{M_{2}}{10^{12} \mathrm{GeV}}\right)\left(\frac{m_{\phi}}{10^{13} \mathrm{GeV}}\right)^{1 / 2}\left(\frac{v_{B-L}}{5 M_{\mathrm{Pl}}}\right)^{-1}\left(1-\frac{4 M_{2}^{2}}{m_{\phi}^{2}}\right)^{3 / 4} .
$$

Here we used $T_{R}=\left(90 / \pi^{2} g_{*}\left(T_{R}\right)\right)^{1 / 4} \sqrt{\Gamma_{\phi} M_{\mathrm{Pl}}}$ and $g_{*}\left(T_{R}\right)=106.75$, where $g_{*}\left(T_{R}\right)$ is the relativistic degrees of freedom in plasma at the temperature $T_{R}$. If the Higgs mode $\phi \rightarrow h h, W W, Z Z$ is the dominant decay channel through eq. (2.3), the reheating temperature can be arbitrarily higher than the above estimate. If $T_{R}$ is higher than $m_{\phi}$, the perturbative analysis of the reheating process becomes unreliable [70, 71]. Therefore, we restrict ourselves to the region of $T_{R}<m_{\phi} \sim 10^{13} \mathrm{GeV}$.

\subsection{Leptogenesis}

For the case where $\phi \rightarrow N_{2} N_{2}$ is the dominant decay mode, the decay of $N_{2}$ can generate $B-L$ asymmetry by leptogenesis. The baryon-to-entropy ratio obtained from the nonthermal leptogenesis is $[72,73]$,

$$
\frac{n_{B}}{s}=-\frac{28}{79} \cdot \frac{3}{2} \cdot \epsilon \cdot \frac{T_{R}}{m_{\phi}}
$$

where $(3 / 2) T_{R} / m_{\phi}$ is the number density $n_{N_{2}} \simeq n_{\phi} / 2$ divided by the entropy density produced by the decay of $\phi$. The $\epsilon$ factor is the magnitude of the CP violation [74]:

$$
\epsilon \simeq-\frac{3}{16 \pi} \frac{\operatorname{Im}\left(y_{\nu} y_{\nu}^{\dagger}\right)_{23}^{2}}{\left(y_{\nu} y_{\nu}^{\dagger}\right)_{22}} \frac{M_{2}}{M_{3}}
$$

for $M_{2} \ll M_{3}$. It is bounded by [51, 75],

$$
|\epsilon| \lesssim\left\{\begin{array}{lll}
\frac{3}{16 \pi} \frac{M_{2}}{\langle H\rangle^{2}}\left(m_{3}-m_{2}\right) \sim 8 \times 10^{-5}\left(\frac{M_{2}}{10^{12} \mathrm{GeV}}\right), & & \text { (Normal) } \\
\frac{3}{16 \pi} \frac{M_{2}}{\langle H\rangle^{2}}\left(m_{2}-m_{1}\right) \sim 2 \times 10^{-6}\left(\frac{M_{2}}{10^{12} \mathrm{GeV}}\right) . & & \text { (Inverted) }
\end{array}\right.
$$

Here, we take $\Delta m_{\odot}^{2}=(0.0086 \mathrm{eV})^{2}$ and $\Delta m_{A}^{2}=(0.048 \mathrm{eV})^{2}[76]$. Therefore,

$$
\left.\frac{n_{B}}{s}\right|_{\max } \simeq\left(\frac{M_{2}}{10^{12} \mathrm{GeV}}\right)^{2}\left(\frac{m_{\phi}}{10^{13} \mathrm{GeV}}\right)^{-1 / 2}\left(\frac{v_{B-L}}{5 M_{\mathrm{Pl}}}\right)^{-1} \times\left\{\begin{array}{l}
1 \times 10^{-10} \text { (Normal) } \\
2 \times 10^{-12} \text { (Inverted) }
\end{array}\right.
$$

For normal hierarchy, compared with the observed baryon-to-entropy ratio, $n_{B} / s \simeq$ $10^{-10}$ [77], we need $M_{2} \gtrsim 10^{12} \mathrm{GeV}$. On the other hand, for inverted hierarchy, we need 
$M_{2} \gtrsim 10^{13} \mathrm{GeV}$ which is on the border of the constraint: $m_{\phi}>2 M_{2}$. In any case, these result justify $M_{3}>m_{\phi}$ which we assumed before.

If the Higgs mode is important, the branching ratio into $M_{2}$ is suppressed, and thus non-thermal leptogenesis becomes difficult. With fixed $m_{\phi}$ from the CMB observations, there is no freedom to make $M_{2}$ larger since the decay into $N_{2}$ becomes kinematically forbidden. Instead, if the reheating temperature is high enough, it is possible to produce $N_{2}$ thermally. The thermal leptogenesis is possible for $10^{9} \mathrm{GeV} \lesssim M_{2} \lesssim T_{R}[75,78]$.

\subsection{Dark matter abundance}

The inflaton also decays into two $N_{1}$ 's. The assumption that $N_{1}$ is long-lived makes it possible to identify this component to be the dark matter of the Universe.

The partial decay width is given by,

$$
\Gamma\left(\phi \rightarrow N_{1} N_{1}\right)=\frac{1}{2} \frac{m_{\phi}}{16 \pi} \frac{M_{1}^{2}}{v_{B-L}^{2}}\left(1-\frac{4 M_{1}^{2}}{m_{\phi}^{2}}\right)^{3 / 2} .
$$

By using the relation $H\left(T_{R}\right) \sim \Gamma_{\phi} \sim T_{R}^{2} / M_{\mathrm{Pl}}$, and $n_{N_{1}} / s \simeq(3 / 2)\left(T_{R} / m_{\phi}\right) \operatorname{Br}\left(\phi \rightarrow N_{1} N_{1}\right)$, we find,

$$
\Omega_{N_{1}}^{\mathrm{NT}} \simeq 0.2\left(\frac{M_{1}}{4 \mathrm{PeV}}\right)^{3}\left(\frac{T_{R}}{3 \times 10^{7} \mathrm{GeV}}\right)^{-1}\left(\frac{v_{B-L}}{5 M_{\mathrm{Pl}}}\right)^{-2}
$$

Here, we used $\Omega_{N_{1}}^{\mathrm{NT}}=\left(M_{1} n_{N_{1}} / s\right) /\left(\rho_{c} / s\right)_{0}$, where $\left(\rho_{c} / s\right)_{0} \simeq 1.8 \times 10^{-9} \mathrm{GeV}$ is the critical density divided by the entropy density today. The contribution from the thermal production from the scattering processes by the $U(1)_{B-L}$ gauge interaction is much smaller such as $[38,39]$,

$$
\Omega_{N_{1}}^{\mathrm{TH}} \sim 10^{-23}\left(\frac{M_{1}}{4 \mathrm{PeV}}\right)\left(\frac{T_{R}}{5 \times 10^{7} \mathrm{GeV}}\right)^{3}\left(\frac{v_{B-L}}{5 M_{\mathrm{Pl}}}\right)^{-4}
$$

This is estimated with the interaction between $N_{1}$ and the Standard Model fermions in plasma through the s-channel exchange of the $U(1)_{B-L}$ gauge boson. We summarize the allowed regions in figure 3 . We see that one obtains the correct amount of the baryon asymmetry and the dark matter abundance at $M_{1} \sim 1 \mathrm{PeV}$ and $M_{2} \sim 10^{12} \mathrm{GeV}$ within the region consistent with the BICEP2 results at the $1 \sigma$ level. The $\mathrm{PeV}$ dark matter opens up an interesting possibility that the high energy neutrinos observed at the IceCube experiment $[40,41]$ are explained by the decay of $N_{1}$, which will be studied in the next section. For a heavier $N_{1}$, we see a region where the thermal leptogenesis works. There, a high enough reheating temperature is realized by the inflaton decay into Higgs fields through the coupling in eq. (2.3).

\section{$5 \quad$ PeV neutrinos as a signal of decaying $N_{1}$}

In this section, we discuss observational signatures of the right-handed neutrino dark matter. As discussed so far, the inflation, the baryon asymmetry and the correct amount of 


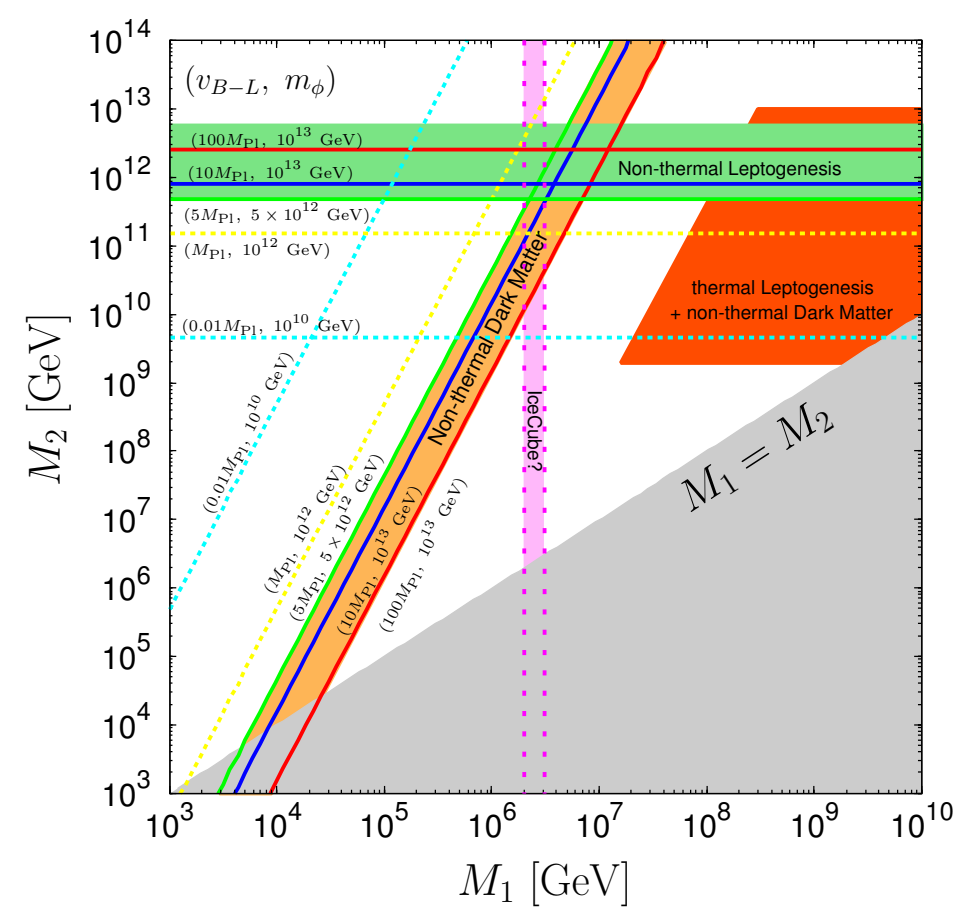

Figure 3. Consistent regions with neutrino masses and cosmological observations. The two shaded regions (green and light orange) are consistent with the BICEP2 at $1 \sigma$ level respectively, and imply that the non-thermal leptogenesis works (green) and the dark matter is explained via the inflaton decay (light orange). Here, we assume normal hierarchy. We also show the mass range of $N_{1}$ favored by the IceCube experiment (pink shaded region). In the dark orange region where thermal leptogenesis is viable, the reheating temperature is treated as a free parameter satisfying $M_{2} \leq T_{R} \leq m_{\phi}=10^{13} \mathrm{GeV}$ with $5 M_{\mathrm{Pl}} \leq v_{B-L}$. A high reheating temperature is realized by the decay into $h h, W W$ and $Z Z$ via the coupling in eq. (2.3).

dark matter can be explained for $M_{1}=\mathcal{O}(1) \mathrm{PeV}$. Since there is no reason to expect that $N_{1}$ is absolutely stable, we have a chance to see high energy cosmic rays produced from the decay of $N_{1}$. It is interesting that the $\mathrm{PeV}$ is indeed the energy region where an excess of high energy neutrinos events are observed at the IceCube experiment. In this section, we discuss the possibility that neutrino excess which is observed at IceCube experiment $[40,41]$ is explained by the decay products of $N_{1}$.

\subsection{The branching fractions of $N_{1}$}

The partial decay widths of $N_{1}$ at tree level are,

$$
\begin{gathered}
\Gamma\left(N_{1} \rightarrow \ell^{-} W^{+}\right)=\Gamma\left(N_{1} \rightarrow \ell^{+} W^{-}\right)=\frac{\left|y_{\nu}^{1 \ell}\right|^{2} M_{1}}{16 \pi}\left(1-\frac{m_{W}^{2}}{M_{1}^{2}}\right)^{2}\left(1+\frac{2 m_{W}^{2}}{M_{1}^{2}}\right), \\
\Gamma\left(N_{1} \rightarrow \nu_{\ell} Z\right)=\Gamma\left(N_{1} \rightarrow \bar{\nu}_{\ell} Z\right)=\frac{\left|y_{\nu}^{1 \ell}\right|^{2} M_{1}}{32 \pi}\left(1-\frac{m_{Z}^{2}}{M_{1}^{2}}\right)^{2}\left(1+\frac{2 m_{Z}^{2}}{M_{1}^{2}}\right) \\
\Gamma\left(N_{1} \rightarrow \nu_{\ell} h\right)=\Gamma\left(N_{1} \rightarrow \bar{\nu}_{\ell} h\right)=\frac{\left|y_{\nu}^{1 \ell}\right|^{2} M_{1}}{32 \pi}\left(1-\frac{m_{h}^{2}}{M_{1}^{2}}\right)^{2} .
\end{gathered}
$$


For $M_{1} \gg m_{W}, m_{Z}, m_{h}$, we can see that $\Gamma\left(N_{1} \rightarrow \ell^{\mp} W^{ \pm}\right): \Gamma\left(N_{1} \rightarrow \nu Z, \bar{\nu} Z\right): \Gamma\left(N_{1} \rightarrow\right.$ $\nu h, \bar{\nu} h) \simeq 2: 1: 1$ due to the equivalence theorem [79-82]. The lifetime of $N_{1}$ for $M_{1} \gg$ $m_{W}, m_{Z}, m_{h}$ is calculated as,

$$
\tau_{N_{1}}=\left(\frac{M_{1}}{4 \pi} \sum_{\ell}\left|y_{1 \ell}\right|^{2}\right)^{-1} \sim 8 \times 10^{28} \mathrm{~s}\left(\frac{M_{1}}{1 \mathrm{PeV}}\right)^{-1}\left(\sum_{\ell}\left|\frac{y_{1 \ell}}{10^{-29}}\right|^{2}\right)^{-1} .
$$

The branching fractions for each lepton family $\operatorname{Br}(\ell) \equiv \operatorname{Br}\left(N_{1} \rightarrow\right.$ $\left.\ell^{\mp} W^{ \pm}, \nu_{\ell} Z, \bar{\nu}_{\ell} Z, \quad \nu_{\ell} h, \bar{\nu}_{\ell} h\right)$ are determined by $y_{\nu}^{1 \ell}$ 's. For each neutrino mass hierarchy, by the assumption of eq. (2.10), $\operatorname{Br}(\ell)$ 's are completely determined by the PMNS matrix,

$$
\begin{array}{lr}
(\operatorname{Br}(e), \operatorname{Br}(\mu), \operatorname{Br}(\tau))=\left(\left|U_{e 1}\right|^{2},\left|U_{\mu 1}\right|^{2},\left|U_{\tau 1}\right|^{2}\right), & \text { (Normal) } \\
(\operatorname{Br}(e), \operatorname{Br}(\mu), \operatorname{Br}(\tau))=\left(\left|U_{e 3}\right|^{2},\left|U_{\mu 3}\right|^{2},\left|U_{\tau 3}\right|^{2}\right) . & \text { (Inverted) }
\end{array}
$$

We take $\sin ^{2} \theta_{12}=0.31, \sin ^{2} \theta_{23}=0.39$ and $\sin ^{2} \theta_{13}=0.02$ [76], then, the numerical values of the branching fraction are given by,

$$
\begin{array}{lr}
(\operatorname{Br}(e), \operatorname{Br}(\mu), \operatorname{Br}(\tau))=(0.68,0.24+0.02 \cos \delta, 0.08-0.02 \cos \delta), & \text { (Normal) } \\
(\operatorname{Br}(e), \operatorname{Br}(\mu), \operatorname{Br}(\tau))=(0.02,0.38,0.60) & \text { (Inverted) }
\end{array}
$$

The branching fractions for normal hierarchy has small dependence on CP-violating phase $\delta$. On the other hand, the branching fractions for inverted hierarchy is completely determined independent of $\delta$.

\subsection{Neutrino flux from decay of $N_{1}$}

We have calculated the energy spectrum of neutrinos $d N_{\nu} / d E_{\nu}$ from decay of $N_{1}$ by using PYTHIA 8.1 [83]. The neutrino spectrum for $M_{1}=2.3 \mathrm{PeV}$ is shown in figure 4 . We have a sharp peak in the neutrino energy spectrum at $E_{\nu}=M_{1} / 2$. In the case of inverted hierarchy, since the fractions of muon and tau are large compared to the normal hierarchy, the number of neutrinos is slightly larger around $E_{\nu} \sim 10^{5-6} \mathrm{GeV}$ due to the decay products of the muons and taus.

As the neutrino travels towards the Earth, the neutrinos change their flavors by the neutrino oscillation according to the following probabilities:

$$
\begin{aligned}
& P\left(\nu_{\ell} \rightarrow \nu_{\ell^{\prime}}\right)=P\left(\bar{\nu}_{\ell} \rightarrow \bar{\nu}_{\ell^{\prime}}\right)=\sum_{i=1}^{3}\left|U_{\ell i} U_{\ell^{\prime} i}\right|^{2} \\
& \simeq\left(\begin{array}{ccc}
0.55 & 0.27+0.02 \cos \delta & 0.18-0.02 \cos \delta \\
0.27+0.02 \cos \delta & 0.36-0.02 \cos \delta & 0.37+0.00 \cos \delta \\
0.18-0.02 \cos \delta & 0.37+0.00 \cos \delta & 0.45+0.02 \cos \delta
\end{array}\right) .
\end{aligned}
$$

In figure 5, we show the energy spectrum of the neutrinos after the oscillation.

We estimate the observed flux of neutrinos on the Earth in the following way. We have two classes of contribution from the decaying dark matter; one is from halo of our galaxy, 


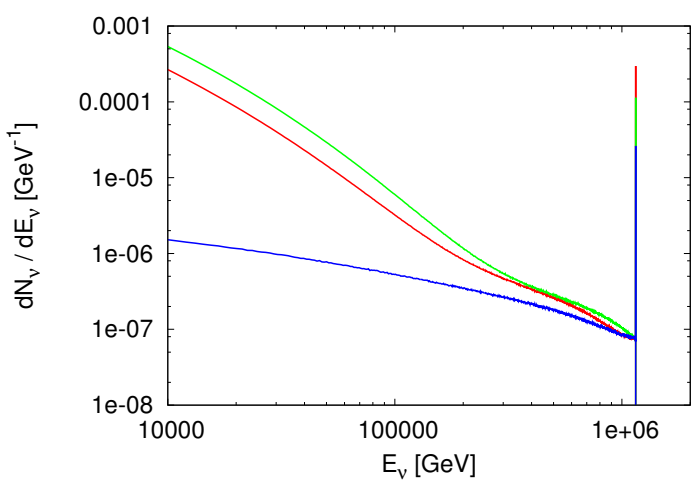

(a) Normal hierarchy $(\delta=0)$

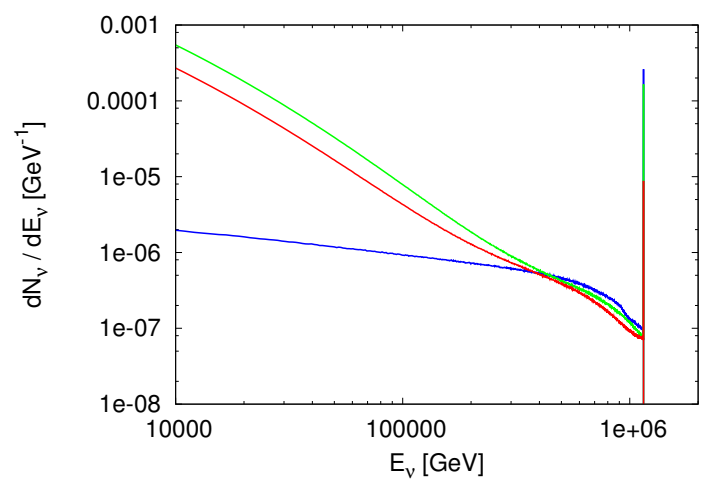

(b) Inverted hierarchy $(\delta=0)$

Figure 4. $d N_{\nu} / d E_{\nu}$ for $M_{1}=2.3 \mathrm{PeV}$ when produced by the decay of $N_{1}$. We take normal hierarchy with $\delta=0$ in left figure and inverted hierarchy in right figure. Red, green and blue lines show the spectrum of $\nu_{e}+\bar{\nu}_{e}, \nu_{\mu}+\bar{\nu}_{\mu}$ and $\nu_{\tau}+\bar{\nu}_{\tau}$, respectively.

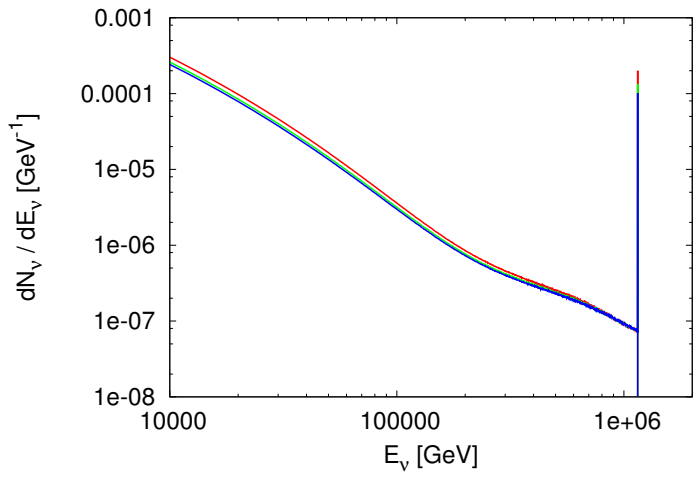

(a) Normal hierarchy $(\delta=0)$

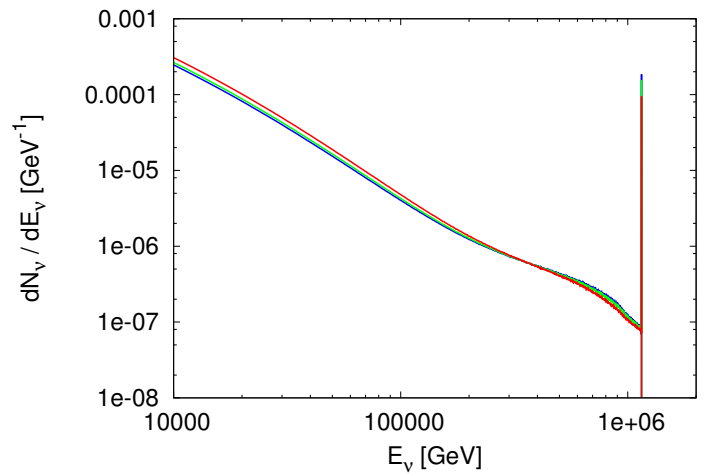

(b) Inverted hierarchy $(\delta=0)$

Figure 5. $d N_{\nu} / d E_{\nu}$ for $M_{1}=2.3 \mathrm{PeV}$ which takes into account the effect of the neutrino oscillation (See eq. (5.9)). We take normal hierarchy in left figure and inverted hierarchy in right figure. In both figure, we take $\delta=0$. Red, green and blue lines show the spectrum of $\nu_{e}+\bar{\nu}_{e}$, $\nu_{\mu}+\bar{\nu}_{\mu}$ and $\nu_{\tau}+\bar{\nu}_{\tau}$, respectively.

and another is from extra-galactic. For a review of the calculation of the neutrino flux, e.g., see ref. [84]. The halo contribution which is averaged over the full sky is proportional to $d N_{\nu} / d E_{\nu}$ :

$$
\frac{d \Phi_{\text {halo }}}{d E_{\nu}}=D_{\text {halo }} \frac{d N_{\nu}}{d E_{\nu}}
$$

where $D_{\text {halo }}$ is determined by the halo density profile $\rho_{\text {halo }}(r)$,

$$
D_{\text {halo }}=\frac{1}{4 \pi} \int_{-1}^{1} d \sin \theta \int_{0}^{2 \pi} d \phi\left(\frac{1}{4 \pi M_{1} \tau_{\mathrm{N}_{1}}} \int_{0}^{\infty} d s \rho_{\text {halo }}(r(s, \theta, \phi))\right) .
$$


The parameter $s$ in the integral of eq. (5.11) is the distance from the Earth, and it is related to the distance $r$ from the galactic center as, $r(s, \theta, \phi)=\sqrt{s^{2}+R_{\odot}^{2}-2 s R_{\odot} \cos \theta \cos \phi}$. Here, $R_{\odot}$ is the distance of the Sun to the galactic center, and we take its value as $8.0 \mathrm{kpc}[85]$. For the calculation of $D_{\text {halo }}$, we adopt the Navarro-Frenk-White (NFW) density profile [86],

$$
\rho_{\text {halo }}(r)=\rho_{\odot} \frac{\left(R_{\odot} / r_{c}\right)\left(1+R_{\odot} / r_{c}\right)^{2}}{\left(r / r_{c}\right)\left(1+r / r_{c}\right)^{2}}
$$

and take $r_{c}=20 \mathrm{kpc}$ and $\rho_{\odot}=0.4 \mathrm{GeV} \mathrm{cm}^{-3}$ [87]. Then, $D_{\text {halo }}$ is calculated as,

$$
D_{\text {halo }}=1.7 \times 10^{-13}\left(\frac{1 \mathrm{PeV}}{M_{1}}\right)\left(\frac{10^{28} \mathrm{~s}}{\tau_{\mathrm{N}_{1}}}\right) \mathrm{cm}^{-2} \mathrm{~s}^{-1} \mathrm{sr}^{-1} .
$$

Extra galactic contribution is redshifted because of the expansion of the Universe. Their contribution is written by,

$$
\frac{d \Phi_{\mathrm{eg}}}{d E_{\nu}}=\left.\frac{\Omega_{\mathrm{DM}} \rho_{c} c}{4 \pi M_{1} \tau_{N_{1}}} \int_{0}^{\infty} \frac{d z}{H(z)} e^{-s\left(E_{\nu}, z\right)} \frac{d N_{\nu}}{d E}\right|_{E=(1+z) E_{\nu}},
$$

where we estimate the integrand just from $z=0$ to $z_{\mathrm{eq}}$ for simplicity and hence also neglect the contribution from the dark matter which had decayed at the radiation dominated era, because we assume the dark matter mass is around $\mathrm{PeV}$ and the energy of neutrino from early universe is too low to explain the IceCube excess. In eq. (5.14), $H(z)=H_{0} \sqrt{\Omega_{\Lambda}+\Omega_{m}(1+z)^{3}}$ is the Hubble expansion rate at the redshift $z$. $c=3.0 \times 10^{10} \mathrm{~cm} \mathrm{~s}^{-1}$ is the speed of light. $s\left(E_{\nu}, z\right)$ is neutrino opacity, which is estimated as $s\left(E_{\nu}, z\right) \sim 10^{-17}(1+z)^{7 / 2}\left(E_{\nu} / 1 \mathrm{TeV}\right)$ for $z<z_{\mathrm{eq}}$ [88]. However, in the present situation, this effect is negligibly small. Then, we take $s\left(E_{\nu}, z\right)$ to be zero for an approximation. For the cosmological parameters, we take $\Omega_{\Lambda}=0.68, \Omega_{m}=0.32, \Omega_{\mathrm{DM}}=0.27$, $H_{0}=67 \mathrm{~km} \mathrm{~s}^{-1} \mathrm{Mpc}^{-1}, \rho_{c}=3 H_{0}^{2} M_{\mathrm{Pl}}^{2} \simeq 4.7 \times 10^{-6} \mathrm{GeV} \mathrm{cm}^{-3}$ and $z_{\text {eq }}=3.4 \times 10^{3}$. These values are derived from the Planck data [77].

Finally, the expected number of events at the IceCube detector per 662 days with given energy is calculated as,

$$
N\left(E_{0} \leq E \leq E_{1}\right)=4 \pi \times 662 \text { days } \times \sum_{\ell=e, \mu, \tau} \int_{E_{0}}^{E_{1}} d E_{\nu}\left(\frac{d \Phi_{\mathrm{halo}}^{\left(\nu_{\ell}+\bar{\nu}_{\ell}\right)}}{d E_{\nu}}+\frac{d \Phi_{\mathrm{eg}}^{\left(\nu_{\ell}+\bar{\nu}_{\ell}\right)}}{d E_{\nu}}\right) \sigma_{\mathrm{eff}}^{\left(\nu_{\ell}\right)}\left(E_{\nu}\right),
$$

where $\sigma_{\text {eff }}^{\left(\nu_{\ell}\right)}$ is the neutrino effective area for each flavor which is given in refs. [41, 89]. The IceCube experiment observed 28 events with deposited energies between 30 and $1200 \mathrm{TeV}$, and the expected number of events from atmospheric muons and neutrinos is $10.6_{-3.6}^{+5.0}$ [41]. For 2.3 PeV dark matter, the total expected number of events for each pattern of the neutrino mass hierarchy is,

$$
\begin{array}{ll}
N\left(30 \mathrm{TeV} \leq E_{\nu}\right)=10.8 \times\left(\tau_{N_{1}} / 10^{28} \mathrm{~s}\right)^{-1}, & (\text { Normal }) \\
N\left(30 \mathrm{TeV} \leq E_{\nu}\right)=13.7 \times\left(\tau_{N_{1}} / 10^{28} \mathrm{~s}\right)^{-1} . & \text { (Inverted) }
\end{array}
$$




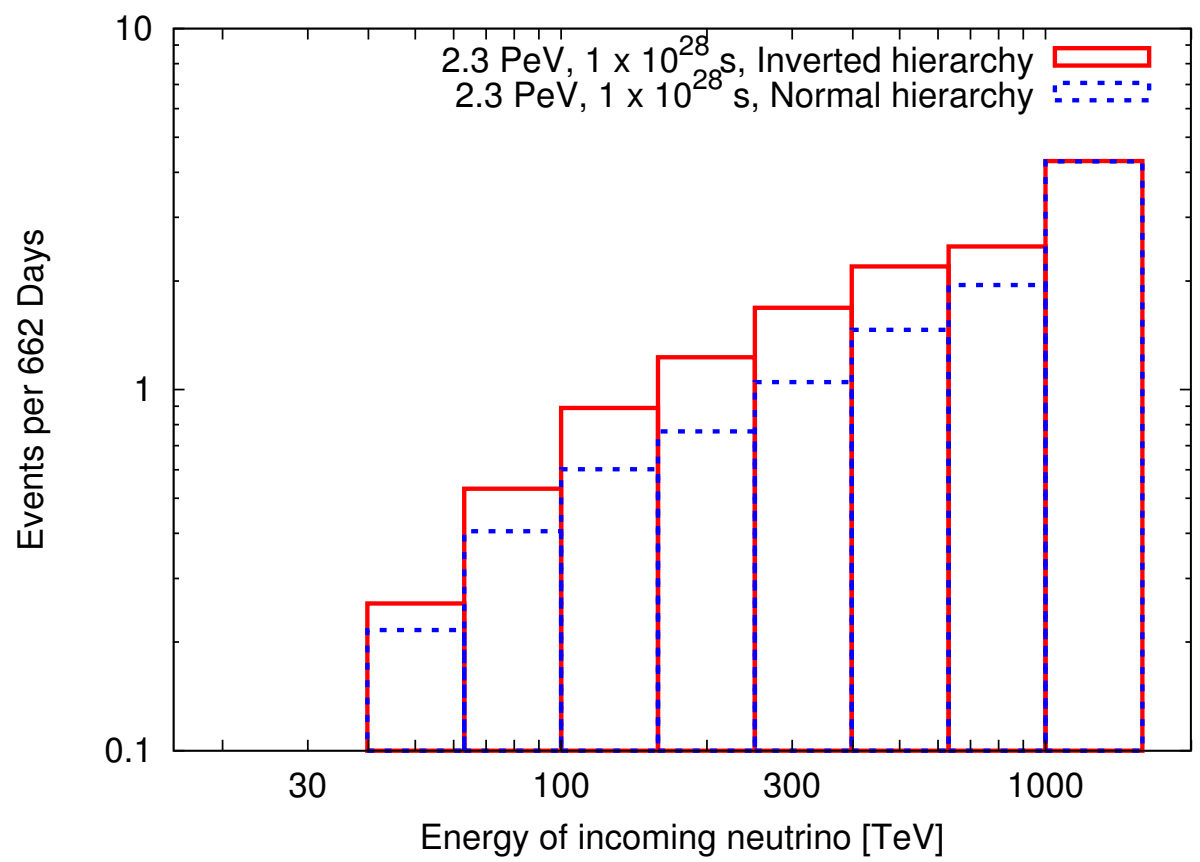

Figure 6. Number of events per 662 days at the IceCube experiment from neutrinos with given energy. For the parameter of $N_{1}$, we take $M_{1}=2.3 \mathrm{PeV}, \tau_{N_{1}}=10^{28} \mathrm{~s}$ and CP-violating phase $\delta=0$. We assume normal hierarchy for dotted blue boxes and inverted hierarchy for solid red boxes.

From this estimate, we see that the total excess can be explained for $\tau_{N_{1}} \simeq 1 \times 10^{28} \mathrm{~s}$ for both normal and inverted hierarchy. We also show the energy distribution of the neutrinos in figure 6. In this figure, we take $M_{1}=2.3 \mathrm{PeV}$ and $\tau_{N_{1}}=10^{28} \mathrm{~s}$.

The IceCube experiment provides the data of the event rate per the deposited energies in the detector in figure 4 in ref. [41]. Note that our results in figure 6 are, in contrast, those for incoming neutrino energies, and thus the deposited ones should be smaller due to escaping neutrinos and muons. One needs to take into account the correction when the shape of the distribution is compared. For $M_{N_{1}}=2.3 \mathrm{PeV}$, the expected number of neutrinos with the energy higher than $1 \mathrm{PeV}$ is,

$$
N\left(1000 \mathrm{TeV} \leq E_{\nu}\right)=4.3 \times\left(\tau_{N_{1}} / 10^{28} \mathrm{~s}\right)^{-1},
$$

for both normal and inverted hierarchy. Thus, by assuming that the deposited energy is equal to that of incoming neutrinos, the two observed neutrino events around $\mathrm{PeV}$ energies can be explained for $\tau_{N_{1}} \simeq 2 \times 10^{28} \mathrm{~s}$. We can expect more sub-PeV events for the inverted hierarchy than the normal hierarchy. Implications from the IceCube experiment will be important to distinguish neutrino models.

\section{Summary}

In this paper, we considered a minimalistic cosmological scenario based on the $U(1)_{B-L}$ extended Standard Model. The model consistently explains the neutrino masses, the in- 
flation, the baryon asymmetry of the Universe and dark matter abundance, which are left unexplained in the Standard Model. If both the baryon asymmetry and the dark matter abundance are explained directly by the inflaton decay, we obtain the mass of $N_{1}$ and the second lightest right-handed neutrino $N_{2}$ to be $1 \mathrm{PeV}$ and $10^{12} \mathrm{GeV}$, respectively.

Interestingly, the mass of $N_{1}, \mathrm{PeV}$, turns out to be the energy scale of the excess of the neutrino events at the IceCube experiment. We see that the $\mathrm{PeV}$ neutrino events can be explained by the decaying $N_{1}$ with its lifetime being $\mathcal{O}\left(10^{28}\right)$ s. Predicted number of neutrinos with sub-PeV energies depends on the neutrino mass hierarchy and the $\mathrm{CP}$ violating phase. Further observations of high energy neutrino events may, in principle, provide information on the flavor structures in the neutrino sector.

If the coupling between the Standard Model Higgs and the $B-L$ Higgs field is significant, the reheating temperature can be higher than the second lightest right-handed neutrino $N_{2}$, depending on the coupling. In such a case, thermal leptogenesis is possible whereas the dark matter should be heavier than $\mathcal{O}(10) \mathrm{PeV}$ to explain the abundance by the inflaton decay.

\section{Acknowledgments}

We would like to thank Aya Ishihara for the explanation of the IceCube experiments. This work is supported by JSPS Grant-in-Aid for Young Scientists (B) (No. 23740165 [RK], No. 25800169 [TH]), MEXT Grant-in-Aid for Scientific Research on Innovative Areas (No. 25105011 [RK]) and JSPS Research Fellowships for Young Scientists [RS].

Open Access. This article is distributed under the terms of the Creative Commons Attribution License (CC-BY 4.0), which permits any use, distribution and reproduction in any medium, provided the original author(s) and source are credited.

\section{References}

[1] A.H. Guth, The Inflationary Universe: A Possible Solution to the Horizon and Flatness Problems, Phys. Rev. D 23 (1981) 347 [inSPIRE].

[2] A.A. Starobinsky, A New Type of Isotropic Cosmological Models Without Singularity, Phys. Lett. B 91 (1980) 99 [INSPIRE].

[3] K. Sato, First Order Phase Transition of a Vacuum and Expansion of the Universe, Mon. Not. Roy. Astron. Soc. 195 (1981) 467 [InSPIRE].

[4] N. Okada and Q. Shafi, Observable Gravity Waves From $U(1)_{B-L}$ Higgs and Coleman-Weinberg Inflation, arXiv:1311.0921 [INSPIRE].

[5] N. Okada, V.N. Senohuz and Q. Shafi, Simple Inflationary Models in Light of BICEP2: an Update, arXiv:1403.6403 [INSPIRE].

[6] A.D. Linde, Chaotic Inflation, Phys. Lett. B 129 (1983) 177 [inSPIRE].

[7] P. Minkowski, $\mu \rightarrow$ er at a Rate of One Out of 1-Billion Muon Decays?, Phys. Lett. B 67 (1977) 421 [INSPIRE]. 
[8] T. Yanagida, Horizontal gauge symmetry and masses of neutrinos, in Proceedings of the Workshop on Unified Theory and Baryon Number of the Universe, eds. O. Sawada and A. Sugamoto (KEK, 1979) p.95

[9] M. Gell-Mann, P. Ramond and R. Slansky, Complex spinors and unified theories, in Supergravity, ed. by D. Freedman and P. Van Nieuwenhuizen, North Holland, Amsterdam (1979), pp. 315-321

[10] S. Glashow, The Future of Elementary Particle Physics, in Quarks and Leptons, Cargèse 1979, eds. M. Lévy et al., Plenum, New York (1980).

[11] R.N. Mohapatra and G. Senjanović, Neutrino Mass and Spontaneous Parity Violation, Phys. Rev. Lett. 44 (1980) 912 [INSPIRE].

[12] J. Schechter and J.W.F. Valle, Neutrino Masses in $S U(2) \times U(1)$ Theories, Phys. Rev. D 22 (1980) 2227 [INSPIRE].

[13] M. Fukugita and T. Yanagida, Baryogenesis Without Grand Unification, Phys. Lett. B 174 (1986) 45 [INSPIRE].

[14] H. Davoudiasl, R. Kitano, T. Li and H. Murayama, The New minimal standard model, Phys. Lett. B 609 (2005) 117 [hep-ph/0405097] [INSPIRE].

[15] Q. Shafi and A. Vilenkin, Inflation with SU(5), Phys. Rev. Lett. 52 (1984) 691 [InSPIRE].

[16] Q. Shafi and V.N. Senoguz, Coleman-Weinberg potential in good agreement with wmap, Phys. Rev. D 73 (2006) 127301 [astro-ph/0603830] [INSPIRE].

[17] C. Destri, H.J. de Vega and N.G. Sanchez, MCMC analysis of WMAP3 and SDSS data points to broken symmetry inflaton potentials and provides a lower bound on the tensor to scalar ratio, Phys. Rev. D $\mathbf{7 7}$ (2008) 043509 [astro-ph/0703417] [INSPIRE].

[18] R. Kallosh and A.D. Linde, Testing String Theory with CMB, JCAP 04 (2007) 017 [arXiv:0704.0647] [INSPIRE].

[19] T.L. Smith, M. Kamionkowski and A. Cooray, The inflationary gravitational-wave background and measurements of the scalar spectral index, Phys. Rev. D 78 (2008) 083525 [arXiv:0802.1530] [INSPIRE].

[20] V.N. Senoguz and Q. Shafi, Chaotic inflation, radiative corrections and precision cosmology, Phys. Lett. B 668 (2008) 6 [arXiv:0806.2798] [InSPIRE].

[21] M.U. Rehman, Q. Shafi and J.R. Wickman, GUT Inflation and Proton Decay after WMAP5, Phys. Rev. D 78 (2008) 123516 [arXiv:0810.3625] [InSPIRE].

[22] M.U. Rehman and Q. Shafi, Higgs Inflation, Quantum Smearing and the Tensor to Scalar Ratio, Phys. Rev. D 81 (2010) 123525 [arXiv: 1003.5915] [INSPIRE].

[23] K. Nakayama and F. Takahashi, Higgs Chaotic Inflation in Standard Model and NMSSM, JCAP 02 (2011) 010 [arXiv: 1008.4457] [INSPIRE].

[24] K. Nakayama and F. Takahashi, Higgs Chaotic Inflation and the Primordial B-mode Polarization Discovered by BICEP2, Phys. Lett. B 734 (2014) 96 [arXiv:1403.4132] [INSPIRE].

[25] K. Nakayama and F. Takahashi, Running Kinetic Inflation, JCAP 11 (2010) 009 [arXiv: 1008.2956] [INSPIRE].

[26] Y. Hamada, H. Kawai, K.-y. Oda and S.C. Park, Higgs inflation still alive, Phys. Rev. Lett. 112 (2014) 241301 [arXiv:1403.5043] [INSPIRE]. 
[27] F. Bezrukov and M. Shaposhnikov, Higgs inflation at the critical point, arXiv:1403.6078 [INSPIRE].

[28] J.L. Cook, L.M. Krauss, A.J. Long and S. Sabharwal, Is Higgs Inflation Dead?, arXiv: 1403.4971 [INSPIRE].

[29] F.L. Bezrukov and M. Shaposhnikov, The Standard Model Higgs boson as the inflaton, Phys. Lett. B 659 (2008) 703 [arXiv:0710.3755] [InSPIRE].

[30] P.J.E. Peebles, Primeval Adiabatic Perturbations: Effect of Massive Neutrinos, Astrophys. J. 258 (1982) 415 [INSPIRE].

[31] K.A. Olive and M.S. Turner, Cosmological Bounds on the Masses of Stable, Right-handed Neutrinos, Phys. Rev. D 25 (1982) 213 [inSPIRE].

[32] S. Dodelson and L.M. Widrow, Sterile-neutrinos as dark matter, Phys. Rev. Lett. 72 (1994) 17 [hep-ph/9303287] [INSPIRE].

[33] X.-D. Shi and G.M. Fuller, A New dark matter candidate: Nonthermal sterile neutrinos, Phys. Rev. Lett. 82 (1999) 2832 [astro-ph/9810076] [INSPIRE].

[34] A.D. Dolgov and S.H. Hansen, Massive sterile neutrinos as warm dark matter, Astropart. Phys. 16 (2002) 339 [hep-ph/0009083] [INSPIRE].

[35] K. Abazajian, G.M. Fuller and M. Patel, Sterile neutrino hot, warm and cold dark matter, Phys. Rev. D 64 (2001) 023501 [astro-ph/0101524] [INSPIRE].

[36] T. Asaka, S. Blanchet and M. Shaposhnikov, The $\nu M S M$, dark matter and neutrino masses, Phys. Lett. B 631 (2005) 151 [hep-ph/0503065] [INSPIRE].

[37] T. Asaka, M. Laine and M. Shaposhnikov, Lightest sterile neutrino abundance within the nuMSM, JHEP 01 (2007) 091 [hep-ph/0612182] [INSPIRE].

[38] A. Kusenko, F. Takahashi and T.T. Yanagida, Dark Matter from Split Seesaw, Phys. Lett. B 693 (2010) 144 [arXiv:1006.1731] [INSPIRE].

[39] H. Ishida, K.S. Jeong and F. Takahashi, Longevity Problem of Sterile Neutrino Dark Matter, Phys. Lett. B 731 (2014) 242 [arXiv:1309.3069] [INSPIRE].

[40] IceCube collaboration, M.G. Aartsen et al., First observation of PeV-energy neutrinos with IceCube, Phys. Rev. Lett. 111 (2013) 021103 [arXiv:1304.5356] [INSPIRE].

[41] IceCube collaboration, M.G. Aartsen et al., Evidence for High-Energy Extraterrestrial Neutrinos at the IceCube Detector, Science 342 (2013) 1242856 [arXiv:1311.5238] [INSPIRE].

[42] B. Feldstein, A. Kusenko, S. Matsumoto and T.T. Yanagida, Neutrinos at IceCube from Heavy Decaying Dark Matter, Phys. Rev. D 88 (2013) 015004 [arXiv:1303.7320] [inSPIRE].

[43] A. Esmaili and P.D. Serpico, Are IceCube neutrinos unveiling PeV-scale decaying dark matter?, JCAP 11 (2013) 054 [arXiv:1308.1105] [INSPIRE].

[44] T. Banks and N. Seiberg, Symmetries and Strings in Field Theory and Gravity, Phys. Rev. D 83 (2011) 084019 [arXiv:1011.5120] [INSPIRE].

[45] M. Berasaluce-Gonzalez, L.E. Ibáñez, P. Soler and A.M. Uranga, Discrete gauge symmetries in D-brane models, JHEP 12 (2011) 113 [arXiv:1106.4169] [INSPIRE].

[46] M. Berasaluce-Gonzalez, P.G. Camara, F. Marchesano, D. Regalado and A.M. Uranga, Non-Abelian discrete gauge symmetries in 4d string models, JHEP 09 (2012) 059 [arXiv:1206.2383] [INSPIRE]. 
[47] R. Blumenhagen, M. Cvetic, S. Kachru and T. Weigand, D-Brane Instantons in Type II Orientifolds, Ann. Rev. Nucl. Part. Sci. 59 (2009) 269 [arXiv:0902.3251] [inSPIRE].

[48] G. 't Hooft, Computation of the Quantum Effects Due to a Four-Dimensional Pseudoparticle, Phys. Rev. D 14 (1976) 3432 [Erratum ibid. D 18 (1978) 2199] [INSPIRE].

[49] G. 't Hooft, How Instantons Solve the U(1) Problem, Phys. Rept. 142 (1986) 357 [INSPIRE].

[50] P.H. Frampton, S.L. Glashow and T. Yanagida, Cosmological sign of neutrino CP-violation, Phys. Lett. B 548 (2002) 119 [hep-ph/0208157] [INSPIRE].

[51] K. Harigaya, M. Ibe and T.T. Yanagida, Seesaw Mechanism with Occam's Razor, Phys. Rev. D 86 (2012) 013002 [arXiv:1205.2198] [InSPIRE].

[52] B. Pontecorvo, Inverse beta processes and nonconservation of lepton charge, Sov. Phys. JETP 7 (1958) 172 [INSPIRE].

[53] Z. Maki, M. Nakagawa and S. Sakata, Remarks on the unified model of elementary particles, Prog. Theor. Phys. 28 (1962) 870 [InSPIRE].

[54] J.A. Casas and A. Ibarra, Oscillating neutrinos and $\mu$ toe, $\gamma$, Nucl. Phys. B 618 (2001) 171 [hep-ph/0103065] [INSPIRE].

[55] A. Ibarra and G.G. Ross, Neutrino phenomenology: The Case of two right-handed neutrinos, Phys. Lett. B 591 (2004) 285 [hep-ph/0312138] [INSPIRE].

[56] A.R. Liddle and D.H. Lyth, Cosmological inflation and large scale structure, Cambridge University Press, Cambridge U.K. (2000).

[57] Planck collaboration, P.A.R. Ade et al., Planck 2013 results. XXII. Constraints on inflation, arXiv:1303.5082 [INSPIRE].

[58] BICEP2 collaboration, P.A.R. Ade et al., Detection of B-Mode Polarization at Degree Angular Scales by BICEP2, Phys. Rev. Lett. 112 (2014) 241101 [arXiv:1403.3985] [INSPIRE].

[59] E. Giusarma, E. Di Valentino, M. Lattanzi, A. Melchiorri and O. Mena, Relic Neutrinos, thermal axions and cosmology in early 2014, arXiv:1403.4852 [INSPIRE].

[60] C.R. Contaldi, M. Peloso and L. Sorbo, Suppressing the impact of a high tensor-to-scalar ratio on the temperature anisotropies, arXiv:1403.4596 [INSPIRE].

[61] M. Kawasaki and S. Yokoyama, Compensation for large tensor modes with iso-curvature perturbations in CMB anisotropies, JCAP 05 (2014) 046 [arXiv: 1403.5823] [INSPIRE].

[62] M. Kawasaki, T. Sekiguchi, T. Takahashi and S. Yokoyama, Isocurvature perturbations and tensor mode in light of Planck and BICEP2, arXiv:1404.2175 [INSPIRE].

[63] V. Miranda, W. Hu and P. Adshead, Steps to Reconcile Inflationary Tensor and Scalar Spectra, arXiv:1403.5231 [INSPIRE].

[64] B. Feng and X. Zhang, Double inflation and the low CMB quadrupole, Phys. Lett. B 570 (2003) 145 [astro-ph/0305020] [InSPIRE].

[65] M. Kawasaki and F. Takahashi, Inflation model with lower multipoles of the CMB suppressed, Phys. Lett. B 570 (2003) 151 [hep-ph/0305319] [INSPIRE].

[66] B. Freivogel, M. Kleban, M.R. Martinez and L. Susskind, Observational Consequences of a Landscape: Epilogue, arXiv:1404.2274 [INSPIRE]. 
[67] R. Bousso, D. Harlow and L. Senatore, Inflation After False Vacuum Decay: New Evidence from BICEP2, arXiv: 1404.2278 [INSPIRE].

[68] H. Murayama, K. Nakayama, F. Takahashi and T.T. Yanagida, Sneutrino Chaotic Inflation and Landscape, arXiv:1404.3857 [INSPIRE].

[69] T. Higaki and F. Takahashi, Natural and Multi-Natural Inflation in Axion Landscape, arXiv: 1404.6923 [INSPIRE].

[70] K. Mukaida and K. Nakayama, Dynamics of oscillating scalar field in thermal environment, JCAP 01 (2013) 017 [arXiv: 1208.3399] [INSPIRE].

[71] K. Mukaida and K. Nakayama, Dissipative Effects on Reheating after Inflation, JCAP 03 (2013) 002 [arXiv: 1212.4985] [INSPIRE].

[72] T. Asaka, K. Hamaguchi, M. Kawasaki and T. Yanagida, Leptogenesis in inflaton decay, Phys. Lett. B 464 (1999) 12 [hep-ph/9906366] [INSPIRE].

[73] T. Asaka, K. Hamaguchi, M. Kawasaki and T. Yanagida, Leptogenesis in inflationary universe, Phys. Rev. D 61 (2000) 083512 [hep-ph/9907559] [INSPIRE].

[74] L. Covi, E. Roulet and F. Vissani, CP violating decays in leptogenesis scenarios, Phys. Lett. B 384 (1996) 169 [hep-ph/9605319] [INSPIRE].

[75] S. Davidson and A. Ibarra, A Lower bound on the right-handed neutrino mass from leptogenesis, Phys. Lett. B 535 (2002) 25 [hep-ph/0202239] [INSPIRE].

[76] Particle Data Group collaboration, J. Beringer et al., Review of Particle Physics (RPP), Phys. Rev. D 86 (2012) 010001 [inSPIRE].

[77] Planck collaboration, P.A.R. Ade et al., Planck 2013 results. XVI. Cosmological parameters, arXiv:1303.5076 [INSPIRE].

[78] W. Buchmüller, P. Di Bari and M. Plümacher, Cosmic microwave background, matter antimatter asymmetry and neutrino masses, Nucl. Phys. B 643 (2002) 367 [Erratum ibid. B 793 (2008) 362] [hep-ph/0205349] [INSPIRE].

[79] J.M. Cornwall, D.N. Levin and G. Tiktopoulos, Derivation of Gauge Invariance from High-Energy Unitarity Bounds on the s Matrix, Phys. Rev. D 10 (1974) 1145 [Erratum ibid. D 11 (1975) 972] [INSPIRE].

[80] C.E. Vayonakis, Born Helicity Amplitudes and Cross-Sections in Nonabelian Gauge Theories, Lett. Nuovo Cim. 17 (1976) 383 [INSPIRE].

[81] B.W. Lee, C. Quigg and H.B. Thacker, Weak Interactions at Very High-Energies: The Role of the Higgs Boson Mass, Phys. Rev. D 16 (1977) 1519 [InSPIRE].

[82] G.J. Gounaris, R. Kogerler and H. Neufeld, Relationship Between Longitudinally Polarized Vector Bosons and their Unphysical Scalar Partners, Phys. Rev. D 34 (1986) 3257 [INSPIRE].

[83] T. Sjöstrand, S. Mrenna and P.Z. Skands, A Brief Introduction to PYTHIA 8.1, Comput. Phys. Commun. 178 (2008) 852 [arXiv:0710.3820] [InSPIRE].

[84] M. Grefe, Neutrino signals from gravitino dark matter with broken R-parity, arXiv:1111.6041 [INSPIRE].

[85] S. Gillessen et al., Monitoring stellar orbits around the Massive Black Hole in the Galactic Center, Astrophys. J. 692 (2009) 1075 [arXiv:0810.4674] [INSPIRE].

[86] J.F. Navarro, C.S. Frenk and S.D.M. White, A Universal density profile from hierarchical clustering, Astrophys. J. 490 (1997) 493 [astro-ph/9611107] [INSPIRE]. 
[87] F. Iocco, M. Pato, G. Bertone and P. Jetzer, Dark Matter distribution in the Milky Way: microlensing and dynamical constraints, JCAP 11 (2011) 029 [arXiv:1107.5810] [INSPIRE].

[88] A. Esmaili, A. Ibarra and O.L.G. Peres, Probing the stability of superheavy dark matter particles with high-energy neutrinos, JCAP 11 (2012) 034 [arXiv:1205.5281] [INSPIRE].

[89] http://www.icecube.wisc.edu/science/data. 